\title{
Lab based surveillance of multidrug resistant Pseudomonas aeruginosa in Cairo university hospitals, Egypt
}

\begin{abstract}
Multidrug resistance is increasingly recorded among $P$. aeruginosa causing health care associated infections (HAI) due to the selective pressure by over-usage of antibiotics. The primary aim of the study was to perform a laboratory based surveillance to investigate the prevalence of $\beta$ - lactamase and $\mathrm{AmpC}$ production and multi-drug resistance among clinical isolates of $P$. aeruginosa causing health care associated infections in Kasr Al Aini School of Medicine and National Cancer Institute, Cairo University, Egypt. $\beta$ - lactamase and AmpC production was tested by the double disc test. Multidrug-resistant P. aeruginosa (MDRPA) were defined as demonstrating resistance to carbapenems, all anti-pseudomonal cephalosporins, amino glycosides and fluoroquinolones. In a six months period, out of 12,524 clinical samples, $2593(20.7 \%)$ P. aeruginosa isolates were identified. Out of these isolates, $1138(43.9 \%)$ were ceftazidime resistant, $138(5.3 \%)$ were ESBL producers, and $661(25.5 \%)$ isolates were confirmed positive for inducible AmpC beta-lactamase. The proportion of multidrug-resistant $P$. aeruginosa was $12.3 \%$. In conclusion, high prevalence of AmpC indicates the necessity of performing routine tests to detect AmpC in ceftazidime resistant $P$. aeruginosa isolates. Lab based surveillance is important to identify the prevalence of MDRPA in order to guide empirical antibiotic therapy and prevent the spread of resistant strains.
\end{abstract}

Keywords: multidrug-resistant $P$. aeruginosa; extended spectrum beta-lactamase; inducible AmpC
Volume 2 Issue 2 - 2015

\author{
Mona wassef, Hadir El Mahallawy, Mai \\ Mahmoud Zafer, Doaa Ghaith, Rasha Abdel \\ Hamid
}

Department of Microbiology and Immunology, Ahram Canadian

University, Egypt

\begin{abstract}
Correspondence: Mai Zafer, Department of Microbiology and Immunology, Faculty of Pharmacy, Ahram Canadian University, 4th Industrial Zone, 6th of October, Giza, Egypt, Tel +2(02)
\end{abstract} 33806765,Email mai_zafer@hotmail.com

Received: January 31, 2015 | Published: April 02, 2015
Abbreviations: MDRPA, multidrug-resistant P. Aeruginosa; ESBL, extended spectrum beta- lactamase; HAIs, health-care associated infections; NCI, national cancer institute; MHA, MullerHinton agar; CLSI, clinical laboratory standards institute; SSIs, surgical site infections; WHO, world health organization; CAZ, ceftazidime; FEP, cefepime; IMP, imipenem; MER, meropenem; PIPTZ, piperacillin/tazobactam; ATM, aztreonam; CIP, ciprofloxacin; GEN, gentamicin; TOB, tobramycin; AMK, amikacin; CXM, cefuroxime; $\mathrm{CEP}$, cefoperazone; $\mathrm{CRO}$, ceftriaxone; $\mathrm{PB}$, polymyxin $\mathrm{B}$

\section{Introduction}

The increasing prevalence of health-care associated infections (HAIs) produced by multidrug-resistant (MDR) P. aeruginosa strains severely compromises the selection of appropriate treatments and is therefore associated with significant morbidity and mortality. ${ }^{1}$ It has been identified as the $2^{\text {nd }}$ most frequent organism causing ventilator-associated pneumonia, the $4^{\text {th }}$ most common causing catheter-associated urinary tract infections, the $5^{\text {th }}$ cause of surgical site infections and the $7^{\text {th }}$ cause of central-line-associated bloodstream infections. ${ }^{2}$

The current increase in incidence of MDR isolates of $P$. aeruginosa raises serious concerns. Multi- drug resistance in P. aeruginosa is defined as the resistance to $\geq 3$ of the following classes of antibiotics penicillin's/cephalosporin's/monobactams, carbapenems, amino glycosides, and fluoroquinolones. Antibiotic selection pressure represents the leading risk factor for MDRPA acquisition. ${ }^{3} P$. aeruginosa strains produce different classes of extended spectrum $\beta$-lactamase (ESBL) that enable bacterium to stand against extended- spectrum cephalosporins, such as cefotaxime, ceftriaxone and ceftazidime and have been reported with increasing frequency. ${ }^{4}$ One of the noteworthy mutation mediated resistance mechanisms are those leading to the repression or inactivation of the carbapenemporinOprD, the hyper production of the chromosomal cephalosporinase $\mathrm{AmpC}$, or the up regulation of one of the several efflux pumps encoded in the P. aeruginosa genome. ${ }^{5}$ Resistance due to plasmid-mediated AmpC enzymes is less common than extended-spectrum beta-lactamase production in most parts of the world but may be both harder to detect and broader in spectrum. ${ }^{6}$ The aim of this study was to perform a laboratory based surveillance to investigate the prevalence of $\beta$-lactamase and $\mathrm{AmpC}$ production and multi-drug resistance among clinical isolates of $P$. aeruginosa causing health care associated infections in Kasr Al Aini School of Medicine and National Cancer Institute (NCI), Cairo University, Egypt.

\section{Materials and methods}

Kasr El Aini School of Medicine and National Cancer Institute are tertiary hospitals belonging to Cairo University, Egypt. The study was approved by Ethics Committee of Cairo University and an informed consent was obtained from all patients receiving treatment and participating in the study. A total of 12,524 clinical samples were collected from patients who were diagnosed to have infections in ICUs and different hospital service units at Kasr El Aini School of Medicine and National Cancer Institute, Cairo University in the period from June 2012 till January 2013. Out of these specimens, 2593 (20.7\%) $P$. aeruginosa isolates were identified and recovered using standard microbiological procedures. The type of specimen and numbers of $P$. 
aeruginosa isolated from each one summarized in (Table 1). Resistance patterns in $P$. aeruginosa were classified according to recently published proposed interim definitions. An isolate was defined as MDR if it was resistant to $\geq 1$ drug in $\geq 3$ categories of drugs. The drugs on which our categorization was based included anti pseudomonal cephalosporin's (ceftazidime [CAZ], cefepime [FEP]), carbapenems (imipenem [IMP], meropenem [MER]), piperacillin/tazobactam (PIPTZ), Aztreonam (ATM), Fluroquinolone (ciprofloxacin [CIP]), and amino glycosides (gentamicin [GEN], tobramycin [TOB], amikacin [AMK]).

Table I Differential numbers of $P$. aeruginosa in relation to different sites of clinical specimens

\begin{tabular}{lll}
\hline Specimen & Total number of specimens (N) & Pseudomonas isolates N (\%) \\
\hline Urinary tract infections (UTIs) & $355 I$ & $823(23)$ \\
Urine & $24 I 2$ & $905(37.5)$ \\
Surgical site infections (SSIs) & & \\
Burns and Skin infections & 1939 & $657(34)$ \\
Wound & 473 & $248(52.4)$ \\
Pus & 4967 & $185(3.7)$ \\
Blood Stream infections (BSIs) & 4835 & $172(3.5)$ \\
Peripheral Blood & 132 & $13(10)$ \\
Central venous Line (CVL) & 1512 & $669(44.2)$ \\
Lower respiratory tract infections & 1320 & $597(45.2)$ \\
Sputum\& Endotracheal & 126 & $71(56.3)$ \\
Pleural fluid & 66 & $1(1.5)$ \\
Bronchoalveolar lavage & 82 & II (13.4) \\
CSF & 12,524 & $2593(20.7)$ \\
\hline Total & &
\end{tabular}

\section{In-vitro antimicrobial susceptibility testing}

Susceptibility of the isolates to the following antibacterial agents was tested by the Kirby-Bauer disc diffusion method using disks (Oxoid ltd., Basin Stoke, Hants, England) on Mueller Hinton agar (Oxoid) and interpreted as recommended by Clinical Laboratory Standards Institute guidelines (CLSI,2010) guidelines: imipenem

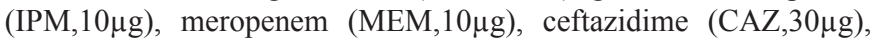
cefoxitin $(30 \mu \mathrm{g})$, cefotaxime $(\mathrm{CTX}, 30 \mu \mathrm{g})$, cefepime $(\mathrm{FEP}, 30 \mu \mathrm{g})$, ciprofloxacin (CIP,5 $\mu$ ), piperacillin/tazobactam (TPZ,100/10 $\mu \mathrm{g}$ ),

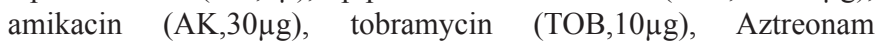

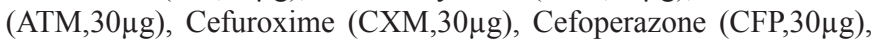
ceftriaxone (CRO, $30 \mu \mathrm{g})$, and Polymyxin B (PB, 300 units).

\section{Extended spectrum $\beta$-lactamase (ESBL) detection}

All $P$. aeruginosa isolates which showed resistance to ceftazidime were evaluated for ESBL production by using the phenotypic confirmatory test. ${ }^{7}$ Briefly, a 0.5 McFarland's suspension of each isolate was spread on a Muller-Hinton agar (MHA) plate and ceftazidime $(30 \mu \mathrm{g})$ and ceftazidime / clavulanic acid $(30 \mu \mathrm{g} / 10 \mu \mathrm{g})$ discs were placed aseptically on the agar plate. A distance of about $15 \mathrm{~mm}$ was kept between the two discs (edge to edge) and the cultures were incubated at $37^{\circ} \mathrm{C}$ overnight. The observation of a $\geq 5 \mathrm{~mm}$ increase in the zone diameter for the antimicrobial agent which was tested in combination with clavulanic acid, versus its zone diameter when tested alone, confirmed the presence of ESBL production by the organism. The increase in the zone diameter was due to the inhibition of the $\beta$-lactamse by clavulanic acid.

\section{Inducible AmpC $\boldsymbol{\beta}$-lactamase detection}

The cefoxitin-cloxacillin disk diffusion test was performed as described by Tan et al. ${ }^{8}$ The test is based on the inhibitory effect of cloxacillin on AmpC. In brief, $30 \mu \mathrm{g}$ cefoxitin disks (Becton Dickinson, Germany) were supplemented with $200 \mu$ g cloxacillin. The test strain was inoculated on Mueller-Hinton agar. The diameters of the cefoxitin inhibition zones were compared with and without cloxacillin; if the difference in inhibition was $\geq 4 \mathrm{~mm}$, the strain was considered positive for AmpC production.

\section{Results}

$P$. aeruginosa were isolated from $20.7 \%$ of samples. The specimens showing the highest isolation of $P$. aeruginosa were from lower respiratory tract infections $(44.2 \%)$ followed by surgical site infections (SSIs), burns \&skin infections $(37.5 \%)$ and urinary tract infections $(23.2 \%)$. Table 1 demonstrates the isolation of $P$. aeruginosa from different sites in relation to total numbers of clinical specimens. The patterns of antibiotic sensitivity of isolated strains of P. aeruginosa are shown in Table 2.

Figure 1 illustrates positive case of AmpC $\beta$-lactamase by the cefoxitin-cloxacillin disk diffusion test. Figure 2 demonstrates the number of ESBL, AmpC and MDR among P. aeruginosa isolates investigated. Of the total $P$. aeruginosa isolates, 1138 showed ceftazidime resistance (43.9\%). A total of $661(25.5 \%)$ isolates were confirmed to be positive for inducible AmpC beta-lactamase. Three hundred and eighteen P. aeruginosa isolates (12.3\%) demonstrated 
resistance to imipenem, meropenem, to all anti pseudomonal cephalosporin's, amino glycosides, fluoroquinolones, and thus, were considered multidrug resistant isolates. Among the 2593 P.aeruginosa isolates, 138 (5.3\%) were ESBL producers.

Table 2 Patterns of $P$. aeruginosa resistance within different types of clinical specimen

\begin{tabular}{|c|c|c|c|c|c|}
\hline Specimen & Total & $\begin{array}{l}\text { Number of } \\
\text { susceptible } P \text {. } \\
\text { aeruginosa } \mathbf{N}(\%)\end{array}$ & $\begin{array}{l}\text { Number of ESBL } \\
\text { producing } P \text {. aeruginosa } \\
\text { N (\%) }\end{array}$ & $\begin{array}{l}\text { Number of Amp } \\
\text { C producing } P . \\
\text { aeruginosa } \mathbf{N}(\%)\end{array}$ & $\begin{array}{l}\text { Number } \\
\text { of MDR P. } \\
\text { aeruginosa N (\%) }\end{array}$ \\
\hline $\begin{array}{l}\text { Urinary tract Infections } \\
\text { (UTIs) Urine }\end{array}$ & 823 & $45 \mathrm{I}(54.8)$ & $94(11.42)$ & $211(25.6)$ & $67(8.1)$ \\
\hline $\begin{array}{l}\text { Surgical site } \\
\text { infections(SSIs) }\end{array}$ & 905 & $552(61)$ & $18(2.0)$ & $222(24.5)$ & $113(12.5)$ \\
\hline \multicolumn{6}{|l|}{ Burns and Skin infections } \\
\hline Wound & 657 & $467(71.1)$ & $18(2.7)$ & $106(16)$ & $66(10.04)$ \\
\hline Pus & 248 & $85(34.3$ & & $116(46.7)$ & $47(19)$ \\
\hline $\begin{array}{l}\text { Lower respiratory } \\
\text { tract infections }\end{array}$ & 669 & $34 I(5 I)$ & $25(3.7)$ & $190(28.4)$ & $113(17)$ \\
\hline Sputum \&endotracheal & 597 & $318(53.2)$ & & $166(27.8)$ & $113(19)$ \\
\hline Pleural fluid & 71 & $23(32.4)$ & $25(35.2)$ & $23(32.4)$ & \\
\hline BAL & 1 & & & $I(100)$ & \\
\hline $\begin{array}{l}\text { Blood stream } \\
\text { infections (BSIs) }\end{array}$ & 185 & $127(69)$ & $22(12)$ & $34(18.3)$ & $23(12.4)$ \\
\hline Peripheral blood & 172 & $125(73)$ & & $24(14)$ & $23(13.4)$ \\
\hline CVL & 13 & $8(61.5)$ & $3(23.1)$ & $2(15.4)$ & \\
\hline CSF & II & $5(45.45)$ & & $4(36.4)$ & $2(18.2)$ \\
\hline
\end{tabular}

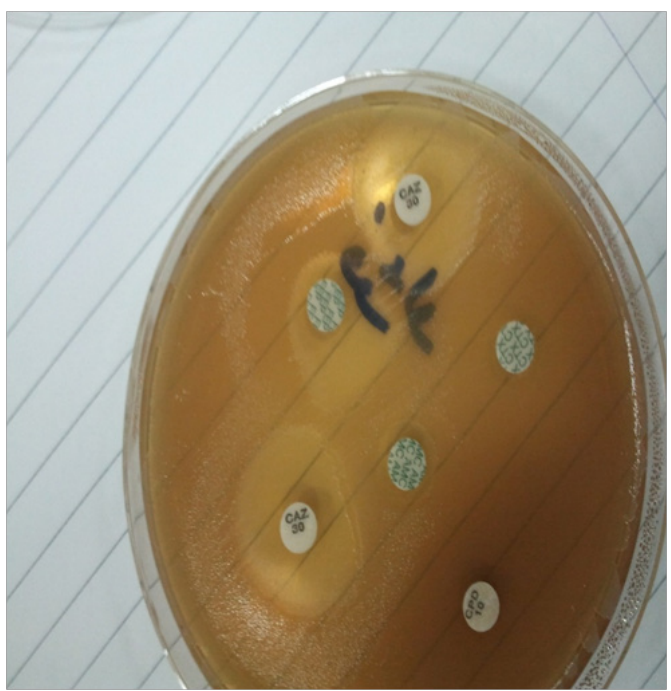

Figure I A positive case of AmpC $\beta$-lactamasesproducer performed by the cefoxitin-cloxacillin disk diffusion test.

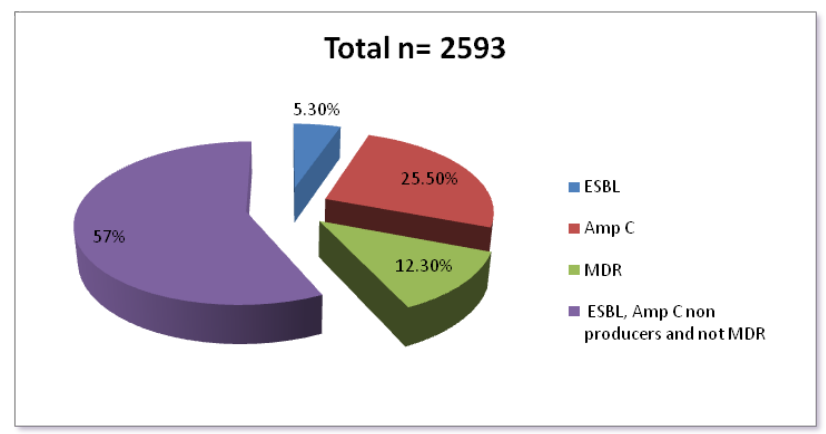

Figure 2 Of the total $P$. aeruginosa tested, 661 were Amp C, 318 were MDR and 138 were ESBL.

\section{Discussion}

$P$. aeruginosa is a leading cause of health-care associated infections ${ }^{9}$ and is extremely difficult to treat due to its high intrinsic and adaptive antibiotic resistance. ${ }^{10}$ Despite the discovery of ESBL, Amp C $\beta$-lactamase and MBL at least a decade ago, there remains 
a low level of awareness of their importance and many clinical laboratories do not undergo routine detection of ESBL \& Amp C $\beta$-lactamase. Failure to detect these enzymes has contributed to their spread and commonly to therapeutic failures.

In the present study a total of 2593 P. aeruginosa strains were isolated in the period from June 2012 till January 2013, accounting for $20.7 \%$ of organisms responsible for health-care associated infections in different hospitals of Kasr Al Aini and National Cancer Institute, Cairo University with $12 \%$ MDR phenotype. These hospitals are tertiary hospitals with expected high rates of complicated cases due to receiving difficult cases from all over the country. Similarly $19 \%$ P. aeruginosa were reported as causes of HAIs in Menofia Hospital University, Egypt with $9.5 \%$ of the isolated P. aeruginosa are corded as MDR. ${ }^{11}$ A figure of $18 \%$ was recorded by Gad in Minia hospitals in Egypt. ${ }^{12}$ It was concluded that MDR P. aeruginosa has emerged in Egypt in recent years and is mainly seen in HAIs. ${ }^{13}$

In the current study, 1138 of $P$. aeruginosa isolated showed resistance to ceftazidime accounting for $43.8 \%$ of the isolates. A high rate of ceftazidime resistance was also reported in a similar Egyptian study in which $45 \%$ of $P$. aeruginosa isolates showed resistance to ceftazidime. ${ }^{14}$ In India, the resistance rate of $P$. aeruginosa to ceftazidime was $53 \% .{ }^{15}$ A much higher rate of ceftazidime resistant $P$. aeruginosa, (91\%) was detected in another part of Egypt. ${ }^{.1}$ Thus, it is evident that high rates of ceftazidime resistance in P. aeruginosa are detected in different areas of Egypt.

$P$. aeruginosa were most commonly isolated from lower respiratory tract infections $(44.2 \%)$, followed by SSIs $(37.5 \%)$, and urinary tract infections $(23 \%)$ in the present study. This may be due to the prolonged hospital stay of patients in the ICU with the need of mechanical ventilation and to the increasing numbers of heart and lung operative procedures done in Kasr Al Aini and NCI hospitals, Cairo University. This is in contrast with the previous studies concluding that $P$. aeruginosa is a nosocomial pathogen often isolated from burn infections. ${ }^{16}$ In the study done by Aly and his colleagues, $P$. aeruginosa accounted for $45 \%$ of infections in the burn unit.

On investigating the rates of $P$. aeruginosa MDR phenotype, inducible Amp C and ESBL production, we found a low prevalence of ESBL producer strains (5.3\%), higher rates of Amp C producer strains (25.5\%); whereas, $12.3 \%$ of isolates were showing an MDR phenotype. In a study done by Zahra and associates (2011) in Iran, low levels of ESBL $P$. aeruginosa producers (9.2\%)was recorded, but with higher levels of the MDR phenotype (30\%). ${ }^{17}$ A high prevalence of ESBL producer strains were reported in a study done in Pakistan accounting for $35.85 \%$ of isolates with $29 \%$ showing an MDR phenotype. ${ }^{18}$ An alarming high prevalence of MDR P. aeruginosa $(52 \%)$ and ESBLs producer strains $(45.6 \%)$ was recently detected in another study done by an Egyptian group of investigators. ${ }^{11} \mathrm{~A}$ high rate of MDR was reported in an Egyptian study, Gad et al., ${ }^{12}$ observed that $36 \%$ of isolated $P$. aeruginosa in their institution were MDR and that $\beta$ - lactamase production was the main mechanism of resistance, as $95 \%$ were ESBL producers.

AmpC producing $P$ aeruginosa were remarkably higher than ESBL producers in our study. Different rates of inducible AmpC producer $P$. aeruginosa strains were recorded in previous studies, varying from $30 \%{ }^{19}$ and up to $60 \% .{ }^{20}$ Being difficult to detect without being alert to its presence, inducible AmpC producers have to be searched for in routine laboratories whenever $P$. aeruginosa is isolated, especially with the increasing rates of ceftazidime resistance. The high rate of inducible AmpC production necessitates exerting efforts to control these types of infections.

The problem of antimicrobial resistance is highlighted by the world health organization (WHO) and combating antimicrobial resistance has been selected as the theme for World Health Day 2011. As the prevalence rates of resistance in gram negative pathogens increase, the treatment options available become limited. Thus, WHO is calling all parties involved in this problem to act and take responsibility for combating antimicrobial resistance. ${ }^{21}$ The rate of MDR P. aeruginosa at our hospitals are not alarming, still awareness, continuous surveillance and antibiotic stewardship are necessary to control and prevent the spread of resistant strains.

In Conclusion, $P$. aeruginosa represented a significant cause of health care associated infections (HAI) in Kasr Al Aini and NCI, Cairo University hospitals. High percent of ceftazidime resistance could be an important cause of treatment failure. AmpC producing $P$. aeruginosa were remarkably higher than ESBL producers detected by double diffusion testing signifying the importance of performing routine tests to detect AmpC in ceftazidime resistant $P$. aeruginosa isolates. Implementing simple and rapid lab screening tests aiming for routine detection of the B-lactamase including the AmpC is needed to monitor the panel of resistance among $P$. aeruginosa. Although MDRPA figures in our hospitals are not alarming, still it is necessary to implement policies to prevent antimicrobial resistance. It is recommended to establish a national program setting on strategies to gather data centrally using sensitive and specific tests to detect mechanisms of resistance and keep a record of the rates of MDR all over Egypt. The optimal contribution of microbiology laboratories is essential to combat the spread of multiply antibiotic-resistant pathogens, which is vital for patient care and safety.

\section{Acknowledgments}

The authors extend their appreciation to the microbiology technicians at Kasr Al Aini School of Medicine and at National Cancer Institute, Cairo University, Reda Zachariah and Ashraf Farag Allah for their cooperation and sincere help.

\section{Conflicts of interest}

Authors declare that there is no conflict of interest.

\section{References}

1. Mesaros N, Nordmann P, Plesiat P, et al. Pseudomonas aeruginosa: resistance and therapeutics options in the turn of the new millennium. Clin Microbiol Infect. 2007;13(6):560-578.

2. Hidron AI, Edwards JR, Patel J, et al. NHSN annual update: antimicrobial-resistant pathogens associated with healthcare-associated infections: annual summary of data reported to the National Healthcare Safety Network at the Centers for Disease Control and Prevention, 20062007. Infect Control Hosp Epidemiol. 2008;29(11):996-1011.

3. Barbier F, Wolf M. Multi-drug resistant Pseudomonas aeruginosa: towards a therapeutic dead end? Med Sci (Paris). 2010;26(11):960-968.

4. Mirsalehian A, Feizabadi M, Nakhjavani FA, et al. Detection of VEB-1, OXA-10 and PER-1 genotypes in extended-spectrum beta-lactamaseproducing Pseudomonas aeruginosa strains isolated from burn patients. Burns. 2010;36(1):70-74.

5. Moya B, Dotsch A, Juan C, et al. Beta-lactam resistance response triggered by inactivation of a nonessential penicillin-binding protein. PLoS Pathog. 2009;5(3):e1000353. 
6. Jacoby GA. AmpC beta-lactamases. Clin Microbiol Rev. 2009;22(1):161-182.

7. Clinical and Laboratory Standards Institute. Performance standards for antimicrobial disk tests. Approved Standards, ( $9^{\text {th }}$ edn), CLSI Document M2-A9: Wayne PA; 2012.

8. Tan TY, Ng LS, He J, et al. Evaluation of screening methods to detect plasmid mediated AmpC in Escherichia coli, Klebsiella pneumoniae, and Proteus mirabilis. Antimicrob Agents Chemother. 2009;53(1):146-149.

9. Picao RC, Poirel L, Gales AC, et al. Diversity of $\beta$-Lactamases produced by ceftazidime-resistant Pseudomonas aeruginosa isolates causing bloodstream infections in Brazil. Antimicrob Agents Chemother. 2009;53(9):3908-3913.

10. Neidig A, Yeung AT, Rosay T, et al. TypA is involved in virulence, antimicrobial resistance and biofilm formation in Pseudomonas aeruginosa. BMC Microbiol. 2013;13:77

11. Mahmoud BA, Zahran AW, Hindawi RG, et al. Prevalence of multidrugresistant $P$ seudomonas aeruginos $a$ in patients with nosocomial infections at a University Hospital in Egypt, with special reference to typing methods. Journal of Virology \& Microbiology. 2013;2013:13.

12. Gad GF, El-Domany RA, Zaki S, et al. Characterization of Pseudomonas aeruginosa isolated from clinical and environmental samples in Minia, Egypt: prevalence, antibiogram and resistance mechanisms. J Antimicrob Chemother. 2007;60(5):1010-1017.

13. Ali AM, Abbasi SA, Ahmed M. Frequency of ES $\beta$ Ls producing nosocomial isolates in a tertiary care hospital in Rawalpindi. Pakistan Armed Forces Medical Journal. 2009;12(3):23-28.
14. Al-Agamy MH, Shibl MA, Zaki AS, et al. Antimicrobial resistance pattern and prevalence of metallo- $\beta$-lactamases in Pseudomonas aeruginosa from Saudi Arabia. African Journal of Microbiology Research. 2011;5(30):5528-5533.

15. Prashan DP, Peerapur BV. ESBL and MBL mediated resistance in Pseudomonas aeruginosa: An emerging threat to clinical therapeutics. Journal of Clinical and Diagnostic Research. 2011;5(8):1552-1554.

16. Pathmanathan SG, Samat NA, Mohamed R. Antimicrobial susceptibility of clinical isolates of Pseudomonas aeruginosa from a Malaysian Hospital. Malays J Med Sci. 2009;16(2):27-32.

17. Zahra T, Moniri R. Detection of ESBLs and MDR in Pseudomonas aeruginosa in a tertiary-care teaching hospital. Iranian Journal of Clinical Infectious Diseases. 2011;6(1):18-23.

18. Ullah F, Malik SA, Ahmed J. Antimicrobial susceptibility and ESBL prevalence in Pseudomonas aeruginosa isolated from burn patients in the north west of Pakistan. Burns. 2009;35(7):1020-1025.

19. Kumar V, Sen MR, Nigam C, et al. Burden of different beta-lactamase classes among clinical isolates of AmpC-producing Pseudomonas aeruginosa in burn patients: A prospective study. Indian J Crit Care Med. 2012;16(3):136-140.

20. Upadhyay S, Sen MR, Bhattacharjee A. Presence of different betalactamase classes among clinical isolates of Pseudomonas aeruginosa expressing AmpC beta-lactamase enzyme. $J$ Infect Dev Ctries. 2010;4(4):239-242.

21. World Health Organization. Antimicrobial resistance. Fact sheet number 194; 2011. 